\title{
ESTUDIO DEL IMPACTO DE LAS NUEVAS FORMAS DE TRABAJO SOBRE LA SALUD DE LOS TRABAJADORES ${ }^{1}$
}

\author{
Maria Cecilia Trionfetti \\ Doctoranda en Sociología del trabajo, Universidad Libre de Bruselas \\ Aline Bingen \\ Profesora de Sociología del trabajo, Universidad Libre de Bruselas \\ Esteban Martinez \\ Profesor de Sociología del trabajo, Universidad Libre de Bruselas
}

\begin{abstract}
El objetivo de este trabajo examinar nuevas formas de trabajo y empleo en el marco de la digitalización de la economía y analizar el impacto de los "usos sociales de los dispositivos digitales en el trabajo" (DUJARIER, 2016), en la salud de los trabajadores. Los resultados obtenidos se basan en el análisis complementario de fuentes bibliográficas y de la encuesta cualitativa realizada en torno a tres tipos de actividades profesionales: las actividades de reparto de mercancías, las actividades de preparación de pedidos en depósitos con el sistema "picking" y las actividades relacionadas con la gestión de expedientes administrativos y bancarios, en el marco de la implementación del modelo de "new ways of working".
\end{abstract}

The aim of this paper is to examine new forms of work and employment in the context of the digitisation of the economy and to analyse the impact of the "social uses of digital devices at work" (DUJARIER, 2016) on workers' safety and health. The paper will present the main finding obtained through a complementary analysis of literature review and a qualitative survey carried out on three types of professional activities: service delivery activities, voice directed warehouse operations and the activities related to the management of administrative and banking files, within the framework of the "new ways of working".

IUSLabor 2/2019, ISSN 1699-2938, p. 32-53

DOI 10.31009/IUSLabor.2019.i02.03

\footnotetext{
${ }^{1}$ El artículo está vinculado al Proyecto de Investigación: Trabajo y Digitalización: el impacto de la economía 4.0 sobre el empleo, las relaciones laborales y la protección social DER2017-82444-R.
} 
Title: Study on the impact of new forms of work on workers' safety and health

Palabras clave: digitalización, condiciones de trabajo, tiempo de trabajo, intensificación, relaciones interpersonales, riesgos profesionales.

Keywords: digitalization, working conditions, working time, intensification, interpersonal relations, professional risks.

\section{Sumario}

1. Introducción

1.1. Perspectiva sociológica y modos de aprehensión de las "nuevas formas de trabajo y empleo"

1.2. Nuevas formas de trabajo y empleo en el contexto de la digitalización de le economía

a. El trabajo móvil basado en las TIC

b. El trabajo en plataformas

2. Metodología

2.1. Objetivos y metodología del proyecto de investigación

2.2. Metodología del estudio cualitativo

3. Los resultados principales del estudio

3.1. La intensificación del trabajo: del trabajo "conectado" al trabajo "invisible"

a. Control del tiempo de trabajo y tareas invisibles

b. Tiempo de trabajo, pausas y descansos

3.2. El uso de dispositivos digitales en el trabajo y sus efectos sobre los tiempos sociales

3.3. Las relaciones interpersonales en el trabajo: distancia real y proximidad virtual

4. Conclusiones

5. Bibliografía 


\section{Introducción}

\subsection{Perspectiva sociológica y modos de aprehensión de las "nuevas formas de trabajo y empleo"}

En los últimos quince años, las diferentes disciplinas han analizado el impacto de los cambios en el mundo del trabajo sobre la salud laboral; ya sea para destacar los efectos de la tercerización de la economía y el desarrollo de nuevas tecnologías en la salud de los trabajadores (CAROLY, 2007); como para demostrar la correlación directa entre la intensificación del tiempo de trabajo y el aumento de los trastornos físicos y psicológicos (DAVEZIES, 2001; GollaC, 2005). Así también dar cuenta del impacto en la salud laboral ocasionado por la implementación del sistema de producción just-intime (DURAND, 2004), o más específicamente, por nuevos modelos de gestión de recursos humanos (DEJOURS, 2003; LINHART 2015).

En el marco de nuestra investigación, la aprehensión de la problemática a través de un enfoque sociológico permite, no sólo abarcar varios niveles de análisis (individuos, empresas, actividades profesionales, configuraciones sociales) sino también problematizar su configuración. Así pues, las nuevas formas de trabajo y empleo pueden abordarse a partir de la articulación de tres enfoques complementarios.

Desde el punto de vista de la organización del trabajo, entendida en términos de modos de división del trabajo y de definición de puestos y funciones, en el tiempo y en el espacio; nos referimos por ejemplo, a los modelos de organización productiva basados en los lemas de lean production y just-in-time (DURAND, 2004; LINHART, 2015), o bien, al modelo de "empresas-red" (LAFUENTE HERNÁNDEZ et al., 2015), donde la organización del trabajo moviliza a un grupo de subcontratistas y trabajadores autónomos, en lugar de contratar directamente al personal necesario para la producción. También podemos destacar los modos de organización del trabajo basados en las "New way of working" (TASKIN, 2010; VENDRAMIN, 2016), un concepto que incluye formas particulares de organización, desde el punto de vista de la gestión del tiempo y del espacio de trabajo. Por último, podemos mencionar a las organizaciones centradas en la autonomía de los trabajadores (VALEYRE, 2007), en este caso el modo de organización del trabajo se basa en reducir el carácter prescriptivo del mismo, aplanar las líneas jerárquicas y responsabilizar al trabajador en función de los objetivos y metas de la empresa. Se trata, por tanto, de una autonomía controlada por los objetivos empresariales.

Desde el punto de vista de la situación laboral, ¿podemos hablar de nuevas formas de empleo? En Bélgica existen dos criterios de distinción: empleados/autónomos; 
privados/públicos. No obstante, las formas contractuales alejadas de la norma social del empleo se han extendido, dando lugar a contratos de duración determinada, trabajo a tiempo parcial o trabajo temporal. A ello se añade el desarrollo de "zonas grises" del mercado laboral, como en el caso de los "falsos autónomos" o de los trabajadores integrados en relaciones multilaterales que se encuentran confrontados a una pluralidad de empleadores. Dicho esto, en general, la situación de los trabajadores asalariados sigue siendo predominante en Bélgica y la proporción de trabajadores autónomos no está aumentando; lo que sí está aumentando es el número de empleados con un estatuto complementario de trabajadores por cuenta propia (MARTínEZ, 2015).

Por último, desde el punto de vista del modo de movilización de la mano de obra (MARTíNEZ, 2015), las nuevas formas de trabajo profundizan una tendencia hacia una polarización entre, por una parte, los trabajadores que disponen de una alto nivel de autonomía, para los que el salario está desconectado de una medición de tiempo y, por otra parte, las categorías de trabajadores más vulnerables (trabajadores menos cualificados con contratos de trabajo a tiempo parcial, trabajadores cedidos por empresas de trabajo temporal), para quienes el tiempo de trabajo se contabiliza estrictamente, pero sólo se pagan las horas de trabajo efectivas.

\subsection{Nuevas formas de trabajo y empleo en el contexto de la digitalización de le economía}

Ahora bien, con el objetivo de delimitar el análisis, nuestro estudio parte de la clasificación de las "nuevas" formas de empleo realizada por Eurofound (2015). El informe presenta, a partir unos sesenta estudios de casos, un panorama general de las tendencias emergentes en términos de "nuevas formas de empleo", su funcionamiento y su impacto en las condiciones de trabajo y el mercado laboral. De acuerdo con nuestro tema de estudio, dos de las nueve formas de empleo identificadas en el informe han sido seleccionadas en función de su relación directa con la utilización de nuevas tecnologías y el fenómeno de "digitalización de la economía"; éstas son: el trabajo móvil basado en las TIC o "trabajo nómada digital" (VENDRAMIN y VALENDUC, 2016) y el "trabajo para plataformas".

a. El trabajo móvil basado en las TIC

El trabajo móvil basado en las TIC (tecnologías de la información y la comunicación) refiere a una forma particular de organización del trabajo desde el punto de vista de la gestión del tiempo y el espacio del mismo. Entre las características más destacadas de esta forma "atípica" de trabajo, el uso de tecnologías móviles (ordenadores portátiles, teléfonos inteligentes, tabletas) y la flexibilidad espacio-temporal son los dos 
componentes principales que transforman radicalmente la organización del trabajo (TASKIN, 2010; Eurofound, 2015; VENDRAMIN et al., 2016). Según la definición adoptada por la Encuesta Europea de Condiciones de Trabajo, el trabajo móvil basado en las TIC se refiere a "trabajadores que pueden trabajar en cualquier lugar y en cualquier momento, con la ayuda de las tecnologías modernas" (Eurofound, 2015). Sin embargo, el informe destaca la diversidad de conceptos utilizados para referirse al mismo fenómeno, a saber: "e-work", "mobile ICT-supported work" (Comisión Europea, 2010), "e-nomades" (Eurofound, 2012) o, tras VendRAmin y VALENDUC (2016), "nomadismo digital".

A diferencia del teletrabajo, la evolución de las TIC permite ir mucho más allá del trabajo a domicilio. Dicho de otro modo, esta nueva forma de organización permite la realización de una actividad independientemente de un tiempo y lugar de trabajo fijos (por ejemplo, en las instalaciones de los clientes, en sitios satélite de la empresa, en el hogar o en espacios públicos). En este sentido, algunos autores refieren a un fenómeno de "desespacialización" y "desterritorialización" del trabajo (TASKIN, 2010), o de "nomadismo digital virtual" (VENDRAMIN et al., 2016) en caso en que los “desplazamientos" para la realización de las actividades sean virtuales.

\section{b. El trabajo en plataformas}

En los últimos diez años, un nuevo modelo económico vinculado a la aparición de las plataformas digitales ha suscitado un gran interés, tanto en el mundo académico y laboral, como a nivel de las instituciones belgas y europeas. En general, los análisis del trabajo para plataformas digitales ponen de relieve el carácter su "innovador", espacialmente con respecto al rol determinante que adquiere los dispositivos digitales en el modo de organización del trabajo (VALENDUC et al., 2016; INRS, 2018). Sin embargo, otros estudios (DE STEFANO, 2016; HuWs et al., 2017) plantean el interés de matizar el carácter disruptivo del fenómeno. Dichos trabajos, sitúan esta nueva forma de trabajo, en la continuidad de los procesos de precarización de las relaciones laborales (DURAND, 2012; BoufFARTIGUE, 2016). En primer lugar, el modo de organización del trabajo en la economía de plataforma contribuye a la elusión de la reglamentación existente en materia de protección de los trabajadores y consumidores (EU-OSHA, 2015 DE SteFAno, 2016; VALENDUC et al., 2016). Asimismo, autores como DeGRYSE (2016) ponen de relieve la emergencia de un "taylorismo digital" o de un "cibertariado" (Huws, 2003) producto de la estandarización y simplificación de las tareas inducidas por los modos de organización del trabajo: "se trata de desglosar el trabajo, o el proceso de producción, en tareas muy pequeñas, simples y repetitivas, que serán propuestas a la "comunidad" de croworkers". A esta tendencia hacia la fragmentación 
del trabajo en micro-tareas se añade, por tanto, el riesgo de sustitución de determinadas formas de trabajo cualificado por formas de trabajo no cualificado (Eurofound, 2015).

\section{Metodología}

\subsection{Objetivos y metodología del proyecto de investigación}

Los datos presentados aquí, son parte de un proyecto de investigación de 10 meses financiado por el Ministerio Belga de Empleo, Trabajo y Concertación Social ${ }^{2}$. La finalidad del proyecto de investigación es la identificación de situaciones de trabajo particularmente impactadas por la digitalización de la economía y el análisis de los "usos sociales de los dispositivos digitales en el trabajo" (DUJARIER, 2016), en la salud de los trabajadores. A nivel metodológico, la primera fase de la investigación incluyó una revisión bibliográfica de artículos científicos y de literatura gris con el fin presentar un panorama de las "nuevas" formas de trabajo y de empleo, en el contexto de la economía digital y de identificar sus efectos en la salud y la seguridad en el trabajo. En la segunda fase de la investigación, se llevó a cabo un análisis cuantitativo basado en datos de la sexta encuesta europea sobre las condiciones de trabajo (EWCS, 2015). Por último, se realizó un estudio cualitativo a través de entrevistas individuales semiestructuradas $(n=11)$, de entrevistas colectivas $(n=4)$ y de la confrontación de los resultados intermedios con un panel de técnicos de prevención de riesgos psicosociales $(n=16)$.

\subsection{Metodología del estudio cualitativo}

Nuestro análisis se basa en los resultados obtenidos a partir de la combinación del examen de la literatura y del análisis temático de los datos de la encuesta cualitativa. Esta última se estructuró en tres etapas:

1. Se realizaron entrevistas individuales semiestructuras de una hora y media a representantes sindicales, consejeros del servicio de estudios de centrales sindicales, inspectores del ministerio de trabajo belga, representantes de asociaciones profesionales de técnicos de prevención de servicios externos y representantes del colectivo de repartidores de plataformas digitales de reparto de comidas a domicilio $(n=11)$.

\footnotetext{
${ }^{2}$ Informe final disponible en línea: http://www.emploi.belgique.be/moduleDefault.aspx?id=47282
} 
2. Se organizaron entrevistas colectivas en torno a tres tipos de actividades profesionales: las actividades de reparto de mercancías, las actividades de preparación de pedidos en depósitos con el sistema picking y las actividades relacionadas con la gestión de expedientes administrativos y bancarios, en el marco de la implementación "new ways of working".

3. Se confrontaron los resultados intermedios con un panel de expertos, compuesto por técnicos en prevención especialistas en aspectos psicosociales $(n=16)$.

Las entrevistas individuales y colectivas se estructuraron en torno al análisis del impacto producido por la utilización de nuevas tecnologías, sobre las cinco dimensiones principales del trabajo (SPF ETC, 2007): la organización, las condiciones, el contenido, el medioambiente y las relaciones interpersonales. El análisis en profundidad de los datos cualitativos se realizó a partir de la codificación y categorización temática de los datos obtenidos en cada grupo de discusión.

\section{Composición de los grupos de discusión:}

\section{- Focus group 1: actividades de reparto o entrega de mercancías}

Cuatro personas participaron en el grupo focal: un delegado sindical de una empresa dedicada a la producción, distribución y venta de bebidas no alcohólicas; un trabajador de una plataforma de entrega de comidas a domicilio; un técnico en prevención de una cooperativa de entrega de pedidos a domicilio y un representante sindical de una central profesional.

- Focus group 2: actividades de preparación de pedidos bajo el sistema de "picking" Cuatro personas participaron del segundo grupo de discusión: una delegada sindical de un distribuidor mayorista de medicamentos, un delegado sindical de una empresa de distribución de alimentos, un técnico de prevención de riesgos laborales de un servicio externo para la prevención y protección de la salud en el trabajo y un médico especialista en salud ocupacional del servicio interno para la prevención y protección de la salud ocupacional de un grupo de distribución de alimentos.

- Focus group 3 \& 4: actividades de gestión administrativa en el marco de la implementación de "Nuevas formas de trabajo" en el sector bancario y de la administración pública

Debido a la cantidad de participantes reunidos, dos grupos de discusión se organizaron y animaron en paralelo. Un total de ocho personas participaron de las entrevistas colectivas: dos delegados sindicales, uno del sector bancario y otro del sector de la administración pública, dos técnicos en prevención de servicios internos de empresas del sector bancario y del sector público, dos técnicos en prevención de servicios externos de prevención y dos gerentes provenientes de cada sector. 


\section{Principales resultados del estudio cualitativo}

Para poner de relieve los factores de riesgos asociados a estas nuevas formas de trabajo, movilizaremos las tres temáticas transversales que surgen del análisis complementario de las fuentes bibliográficas y de los resultados de la encuesta cualitativa: a) la intensificación del trabajo, b) los tiempos sociales, y c) las relaciones interpersonales en el trabajo.

\subsection{La intensificación del trabajo: del trabajo "conectado" al trabajo "invisible"}

a. Control del tiempo de trabajo y tareas invisibles

El uso de la tecnología digital en el trabajo modifica las formas en las que el mismo es prescripto. Así pues, el hecho que la utilización de dispositivos digitales permita que el tiempo asignado a las tareas y a la definición de normas de productividad sea calculado haciendo abstracción de las condiciones reales de ejercicio de la actividad, juega un rol determinante en el aumento de la intensidad del trabajo y, por tanto, en la exposición de los trabajadores a peligros y riesgos laborales generados por esta situación (GoLLAC et al., 1996).

Si nos referimos a los resultados obtenidos en nuestro trabajo de campo, tanto los trabajadores que disponen de un escaso nivel de autonomía (repartidores o preparadores de pedidos) como aquellos considerados altamente autónomos en la gestión del tiempo y del lugar de trabajo (trabajadores móviles), ponen de manifiesto la forma en la que el uso de estas nuevas tecnologías como dispositivos de gestión contribuye a ampliar la distancia que existe entre el "trabajo prescripto" y el "trabajo real" (ODONNE, 2015).

En el caso de la preparación de pedidos, la descripción de la actividad realizada por los trabajadores da cuenta de dos características principales del modo de trabajo bajo el sistema de reconcomiendo vocal: un escaso nivel de autonomía y un control estricto del tiempo y del contenido del trabajo. De hecho, el control jerárquico se efectúa a través de la utilización de un sistema de reconocimiento vocal que ordena a los trabajadores dónde deben estar, qué es lo que deben hacer y cómo deben hacerlo. Dado que los modos de funcionamiento son dictados por este dispositivo digital, cada una de las tareas realizadas por los trabajadores debe seguir el itinerario de trabajo impuesto por el mismo. En términos de riesgos profesionales, uno de los problemas principales, es el del bajo margen de decisión y autonomía (DAVEZIES, 2008).

La falta de visibilidad global sobre el circuito de producción no solamente reduce el control de los operarios sobre el proceso laboral y los contenidos del trabajo, sino que 
también anula la capacidad de anticipación de los preparadores. La imposibilidad de cambiar el orden de preparación de los pedidos frente a un problema técnico, la no toma en cuenta de las condiciones del entorno físico de trabajo, o incluso, las interrupciones en la producción aparecen en este contexto como los principales factores organizacionales causantes de la sobrecarga física y mental de los trabajadores. Como señala una de las trabajadoras:

"No podemos tomar ninguna decisión. Usted tiene la máquina que le dice "haga esto", no se puede pensar, decidir, ni nada. (...) Solo trabajamos con una palabra escrita en mayúscula en nuestra cabeza: PRODUCTIVIDAD. Cuando el picking no funciona, todo lo que te dices es "estoy perdiendo líneas". Así es como trabajamos. Esta palabra está presente todo el tiempo y sólo piensas en la cantidad de líneas que no vas a hacer, porque sabes que al final del día, si no alcanzas los objetivos, serás convocado por el supervisor. Así que no tienes otra opción. No se puede decir: "Hoy voy a hacer esto en lugar de eso", no, ni bien comienza la jornada te pones el auricular, te conectas al sistema y hasta el último momento del día sólo haces lo que la máquina te ordena que hagas" (Myriam, preparadora).

A este respecto, es interesante observar que otro de los entrevistados ahonda en este mismo sentido refiriéndose, más precisamente, al sentimiento de angustia e incertidumbre ocasionado por la incapacidad de anticipación de la carga de trabajo real que deberá realizar.

"Una vez que el repartidor comienza a trabajar para la plataforma, no tiene la posibilidad de elegir casi nada. Es decir, en ese momento debe estar en su zona de trabajo esperando un nuevo pedido, sin saber si ese día hará una, cinco, diezo ninguna entrega" (Antoine, repartidor)

Puesto que, en el caso de los trabajadores de plataformas, todos los procesos de trabajo son coordinados a través de una aplicación que reemplaza la figura del supervisor, los repartidores no disponen de prácticamente ninguna capacidad de modificar o de anticipar el orden prescripto para la realización de tareas. La aplicación indica al repartidor la dirección del restaurante dónde debe recoger el pedido y luego, una vez que la acción ha sido validada por intermedio de la aplicación, el repartidor recibe la dirección del cliente final. Asimismo, la aplicación permite, por ejemplo, evaluar la productividad de los repartidores a partir del cálculo de la velocidad media del conjunto de los trabajadores. Sin embargo, como ha indicado uno de los repartidores, la evaluación se realiza sin tener en cuenta ningún otro aspecto de la situación de trabajo: 
"Por medio del sistema de geolocalización la aplicación puede conocer la velocidad media alcanzada durante el recorrido, pero si el tiempo de espera en el restaurante es muy largo, si el cliente tarda en bajar a recibir el pedido, o simplemente si tenemos retraso porque hay un problema de la calle.... iLa aplicación no lo tiene en cuenta! ¡Es tiempo no remunerado!" (Claude, repartidor)

Por otro lado, en el caso de los trabajadores móviles dentro del sector de la administración pública o del sector bancario, el uso de dispositivos digitales viabiliza un mayor margen de decisión y de autonomía en la gestión del tiempo y del lugar de trabajo. No obstante, la incidencia de la digitalización en la intensificación del trabajo y el aumento de los riesgos relacionados con el "estrés de la auto-organización" (PoPMA, 2013) también aparecen como una preocupación transversal de los participantes entrevistados.

Trabajadores y técnicos en prevención manifiestan los límites de estas nuevas formas de trabajo, al poner de relieve las tensiones generadas por lógicas de gestión contradictorias entre, por un lado, la promoción de la autonomía y, por el otro, la disponibilidad permanente que conlleva el modo de trabajo en " $\mathrm{H}-24$ ".

“ ¿La forma en que trabajamos en $\mathrm{H}-24,7 / 7$ es horrible! Significa que si recibes un mensaje de texto o un email a las 11 ¿tienes que responder? ¡Son muchas las personas que dejan el teléfono profesional encendido durante la noche!" (Laure, técnica de prevención del sector público)

La acumulación de demandas y exigencias, las presiones de tiempo y de resultados, generan tensiones de distinto tipo y situaciones estresantes obligándolos a aumentar la carga de trabajo real. Tal y como señala CAROLY (2007) el uso intensivo de nuevas tecnologías genera dos tipos de sobrecargas, por un lado, una "sobrecarga de información" relacionada con el aumento de la cantidad de información recibida y, por otro, una "sobrecarga de comunicación" asociada al imperativo de "adaptar el mensaje a la diversidad de los interlocutores y de responder a señales diferentes de manera constante." Asimismo, los trabajadores manifiestan estar expuestos al estrés de la autoorganización como resultado de un cambio de prácticas de gestión orientada hacia la responsabilización del trabajador y un debilitamiento del apoyo social recibido por la jerarquía.

"La filosofía de nuestra empresa es: el trabajador tiene autonomía y debe poder ir con su jefe y no esperar pasivamente a que él venga a decirle que debemos cambiar los objetivos (...). Hay una supuesta autonomía del trabajador, pero en 
la práctica jeso no es real! porque hay un marco más estricto, tenemos plazos mucho más importantes y al final multiplicamos los medios y las personas a quienes debemos rendir cuentas." (Michelle, trabajadora del sector bancario)

La descripción realizada por la trabajadora refiere a una situación de "autonomía paradójica" dado que "la conexión permanente con la empresa da paso a una autonomía debilitada donde la gestión de las tareas y la organización del trabajo se llevan a cabo con latitud, mientras se inscriben en un marco de tiempo vinculante, incluso alienante" (ANDONOVA, 2016).

b. Tiempo de trabajo, pausas y descansos

Con respecto a la relación entre la introducción de dispositivos digitales en el trabajo y los usos del factor tiempo como elemento de organización productiva, en todos los casos analizados la eliminación de la "porosidad" del tiempo de trabajo, es decir de las pausas, de los momentos personales o la sociabilidad habitualmente presentes en el trabajo, se traducen en una intensificación del trabajo.

Tanto en la preparación de pedidos guiados por el sistema de picking como en la entrega de comidas a domicilio gestionadas a través de una aplicación móvil, los ritmos y cadencias de trabajo son dictados por dispositivos digitales. Dichos modos de organización del trabajo juegan un rol determinante en la limitación del margen de maniobra de los trabajadores sobre el contenido del trabajo, así como también en la reducción y/o eliminación de los tiempos de recuperación y descanso (DAVEZIES, 2008; VALENDUC et al., 2016; INRS, 2018). El uso de las pausas para compensar retrasos o para solucionar acontecimientos imprevistos, no cubiertos por los dispositivos digitales, forma parte del cotidiano de los preparadores de pedidos con picking y de los repartidores de las plataformas de entrega de comidas a domicilio.

En ambos casos, aparece como espacialmente importante el uso del factor tiempo como herramienta disciplinaria. Así pues, la implementación de sistemas digitalizados de medición diaria de resultados sumada a condiciones de empleo precarias agrava, objetivamente, la exposición a riesgos laborales (IRANI et al., 2013; ROSENBLAT et al., 2016; Degryse, 2016; Huws et al., 2017). El riesgo de despido, el anhelo de un contrato de trabajo por tiempo indeterminado o de aumentar sus ingresos a fin de mes, constituyen factores organizacionales con una fuerte incidencia sobre el aumento de la presión física y mental en el trabajo. 
Cabe descartar, que, en el caso de los trabajadores de plataformas, a esta forma de intensificación del trabajo, se suma el no reconocimiento de los repartidores como empleados de la plataforma y la ausencia de una remuneración horaria garantizada.

En nuestras entrevistas, los repartidores ponen de relieve la relación directa que existe entre el sistema de remuneración por pedidos y el aumento de su exposición a múltiples riesgos, principalmente accidentes de tránsito producto, por un lado, de no respetar el código de circulación, con el afán de maximizar el tiempo de reparto de cada pedido y, por otro, del agotamiento físico ocasionado por las extensas jornadas de trabajo. Dicho de otro modo, cuantas más horas trabajen, menos repartos rechacen y más rápido entreguen el pedido, mejor será la posición del trabajador en el ranking interno y la posibilidad de trabajar durante las horas de alta demanda.

"Cuando te pagan par la cantidad de envíos realizados, la idea de hacer una pausa ni siquiera se te cruza por la cabeza... ni siquiera lo piensas" (Julien, repartidor).

Asimismo, en el caso de los trabajadores móviles del sector bancario y de la administración pública, las prácticas de trabajo flexibles, respecto del tiempo y del lugar de trabajo, también afectan los tiempos de pausa y de descanso. En lo que respecta al bienestar en el trabajo, la mayoría de los entrevistados tienden a matizar las ventajas de trabajar en cualquier momento y en cualquier lugar. Al respecto, técnicos en prevención y trabajadores mencionan con frecuencia dos factores organizacionales estrechamente vinculados a la introducción de dispositivos digitales en el trabajo: el incremento de la disponibilidad temporal y la desmaterialización de los lugares de trabajo.

Dado que el contenido del trabajo de los trabajadores móviles se vuelve parcialmente invisible, la presión social por demostrar los resultados obtenidos a distancia incita a los trabajadores a aumentar la disponibilidad respecto a los colegas y al supervisor (PoPMA, 2013; MetTling, 2015; Eurofound, 2015, 2017). Como comenta una técnica en prevención especialista en aspectos psicosociales:

"Si un jefe de departamento o el director general le envía emails a las 22hs, para un colaborador esto significa implícitamente: "Tengo que trabajar en ese momento" (Laure, técnica en prevención, sector de la administración pública).

En este mismo sentido apunta la intervención de otro técnico en prevención:

"El gerente tiene una posición que le permite, en función de sus recursos, decir que "no". Pero esta no es la realidad de la mayoría de los trabajadores, que no 
tienen la posibilidad de decir abiertamente "no" a su supervisor"." (François, técnico de prevención de un servicio externo).

A esta forma de intensificación se suma la reducción de las pausas, de los momentos de descanso y de sociabilidad, habitualmente presentes en el trabajo, producto de la creciente desmaterialización de los lugares de trabajo.

\subsection{El uso de dispositivos digitales en el trabajo y sus efectos sobre los tiempos sociales}

Pesa a que las actividades analizadas comportan un importante grado de heterogeneidad respecto del contenido de las tareas realizadas, de las condiciones de empleo y del nivel de autonomía sobre la gestión del tiempo y del lugar de trabajo; cabe señalar que, los diferentes actores entrevistados ponen en evidencia situaciones similares en términos de la sobrecarga mental ocasionada por el sentimiento de conectividad permanente.

En el caso de los preparadores de pedidos, el desgaste mental que se produce en este tipo de actividades está asociado a exigencias generadas por el carácter rutinizado, monótono y repetitivo de la actividad. Al referirse a la estandarización de procesos de trabajo, los entrevistados evocan un sentimiento de alienación producto de la "robotización" de los gestos y la repetición constante de códigos durante la jornada laboral. De hecho, la característica principal del sistema picking, es la capacidad de control de cada gesto y movimiento del trabajador. El control del ritmo de trabajo se efectúa a través de un sistema de gestión de almacén que convierte la información relativa a la preparación del pedido en comandos de voz, que el preparador escucha en sus auriculares y que debe ejecutar y validar antes de poder pasar a la siguiente etapa, es decir, en todo momento el trabajador debe responder a une secuencia de instrucciones codificadas. Las situaciones de la vida cotidiana puestas de manifiesto por los trabajadores, permiten ilustrar a las claras la forma en que, la carga mental ocasionada por el uso del dispositivo trasciende las fronteras del ámbito laboral, penetrando paulatinamente en vida privada de los trabajadores:

"Cuando estamos en casa, nos sucede muchas veces de ir a la tienda, de caminar entre las góndolas de pronto y decir" QKZ "[código de un artículo], de estar hablando con un amigo y frente a una pregunta decir" repetir ", o también miras televisión y dices números. ¡Les juro que no estoy bromeando!" (Myriam, preparadora).

"¡Es verdad! Lo hacemos tanto, que incluso cuando no estamos trabajando, sólo tenemos códigos en la cabeza" (Robert, preparado). 
En el caso de los trabajadores de reparto, en particular aquellos que trabajan para plataformas digitales, la ausencia de regulación del tiempo de trabajo junto con el sistema de remuneración por entrega realizada y la utilización de sistemas de evaluación de rendimiento individual, reduce el control sobre el contenido del trabajo al mismo tiempo que aumenta la dependencia de los trabajadores hacia la plataforma. En este sentido, los repartidores entrevistados, refutan el mensaje transmitido por las plataformas que subrayan la flexibilidad y autonomía de la que gozan los repartidores que quieren formar parte de la actividad ("una actividad flexible", "eliges cuando trabajar"). Por el contrario, los trabajadores ponen de relieve la forma en la que dichas ventajas proporcionadas por la plataforma se convierten rápidamente en una fantasía que poco tiene que ver con sus condiciones de trabajo reales. Tal como describe uno de los repartidores, la sujeción de la vida cotidiana a horarios impredecibles o atípicos es una condición sine qua non para que la actividad sea rentable:

"Es un trabajo sin restricciones ipero no precisamente para nosotros...para la plataforma! La flexibilidad sólo funciona en una dirección.... La latitud ya no existe porque los trabajadores tienen que trabajar en los turnos donde hay pedidos, y los momentos donde hay pedidos son los fines de semana y en la noche. Además, existe un sistema de prioridades, lo que significa que aquellos que han hecho la mayor cantidad de entregas, podrán tener acceso prioritario a los turnos, pero para tener esta prioridad, tendrán que trabajar en el momento en que LA PALTAFORMA los necesite" (Julien, repartidor).

En el caso de los trabajadores móviles, la apreciación sobre el efecto de nuevas formas de trabajo para conseguir una conciliación entre la vida laboral y la vida personal muestra resultados ambiguos. Mientras que para algunos, la flexibilidad espaciotemporal permitida por el uso de nuevas tecnologías favorece una conciliación entre vida privada y profesional (mediante la reducción del tiempo de desplazamiento entre el domicilio y el lugar de trabajo o la posibilidad de asumir las responsabilidades familiares sin tener la necesidad de trabajar a tiempo parcial), para otros, esta tendencia hacia la realización de la tareas independientemente de un lugar y un tiempo de trabajo, fijo conlleva necesariamente el riesgo de intensificación del trabajo. En este sentido, la extensión del uso de dispositivos móviles, así como la expansión de Internet, refuerzan la posibilidad de una conectividad permanente, la prolongación de la jornada de trabajo, en ocasiones hasta altas horas de la noche o durante los fines de semana, y por tanto, los riesgos laborales asociados al incremento de la disponibilidad temporal de los trabajadores (PoPMA, 2013; METTLING, 2015; Eurofound, 2015, 2017), tal y como lo expresan una trabajadora del sector bancario y una técnico de prevención: 
"No hay una línea clara, la gente piensa que puede administrar su propio tiempo y, de hecho, se convierten en adictos... de hecho, ellos también se fijan metas inalcanzables" (Michelle, trabajadora, sector bancario).

"El último análisis de riesgos psicosociales que hicimos hace dos años, constatamos que, en ciertas direcciones del ministerio, una gran proporción de personas trabaja más del $136 \%$ del tiempo de trabajo, lo que quiere decir que todo este trabajo, lo hacen los fines de semana o la noche" (Laure, técnica de prevención, sector de la administración pública).

\subsection{Las relaciones interpersonales en el trabajo: distancia real y proximidad virtual}

En cuanto al impacto de las nuevas formas de trabajo sobre las relaciones interpersonales, nos interesa destacar, a partir nuestro trabajo de campo, la manera en que la utilización de dispositivos digitales modifica las relaciones con la jerarquía y con los colegas, como así también su incidencia en la satisfacción por el trabajo y la construcción del colectivo laboral.

Durante las entrevistas colectivas, los trabajadores móviles manifiestan ciertas inquietudes vinculadas a la "virtualización de las relaciones profesionales" (DEGRYSE, 2016). El uso de nuevas tecnologías de la información permite diversificar los canales de comunicación e incrementar los intercambios frecuentes entre los equipos y la jerarquía independientemente de la ubicación y del horario de trabajo. Sin embargo, dichas innovaciones pueden constituir factores de riesgos psicosociales (CAROLY, 2007; 2009; Popma, 2013; Degryse, 2016; Andonova, 2016). De hecho, la problemática del aislamiento social y el debilitamiento del colectivo de trabajo son percibidas como algunas de las principales dificultades de estas nuevas formas de trabajo. La gran mayoría de los entrevistados manifiestan que el hecho de trabajar en cualquier lugar y en todo momento reduce, in fine, las posibilidades de intercambio interpersonal en torno al espacio de encuentros informales y dificultan la organización de actividades colectivas. El problema de la gestión del ruido y las dificultas de concentración en "open-spaces" mal diseñados también son identificados por los técnicos en prevención como otro de los aspectos que afectan las relaciones personales en el trabajo. Esta situación frecuentemente incrementa el aislamiento de los trabajadores, ya sea a través de un aumento de los días de teletrabajo o de una tendencia a retirarse de los espacios de trabajo compartidos. Como señalan dos técnicas de prevención:

“En los servicios donde los trabajadores realizan un trabajo a distancia más de tres veces por semana, hay muchos problemas de cohesión de grupo. Por ejemplo, cuando tienes un problema con tu jefe o un colega, muchas veces la 
manera de "resolverlo" es no verlo durante un mes" (Anne, técnica de prevención, sector de la administración pública).

"En todo lo relacionado con el aprendizaje, el intercambio de conocimiento o el apoyo entre colegas, el trabajo a distancia es un problema. A medida que se pierde el contacto personal cotidiano, aumenta la sensación de falta de apoyo social dentro de los equipos de trabajo" (Caroline, técnica de prevención, sector de la administración pública).

Por otra parte, para los preparadores de pedidos, la escasa posibilidad de comunicación con la jerarquía y su negativa a tener en cuenta la carga real de trabajo se traduce en sentimientos de falta de reconociendo en el trabajo. El desconocimiento de las realidades laborales también resulta ser uno de los principales aspectos reportados por los repartidores.

"En términos de comunicación con la plataforma, prefiero buscar el nombre de la persona en un edificio de cien personas, antes que tener que llamar al servicio al cliente. Hace tiempo que el servicio fue deslocalizado a Madagascar, entonces, cuando llamas y dices: "Estoy en Bruselas, en esta zona" y la respuesta es "dónde está Bruselas?" ya sabes que vas a perder al menos quince minutos antes de obtener un solución a tu problema. (...) Si tienes problemas con la aplicación, el contacto con la plataforma casi siempre es inútil. Una vez me dieron un número de contacto en Inglaterra. Luego de varios intentos, recibí una respuesta automática diciendo que iban a tratar de solucionarlo y nunca más recibí una respuesta... Tuve que borrar la aplicación, recargarla y durante tres o cuatro días no funcionó...no puede trabajar" (Claude, repartidor).

Como mencionamos con anterioridad, el trabajo prescripto a través de una aplicación móvil sumado a la escasa o nula posibilidad de comunicación con la plataforma genera en los repartidores un sentimiento de irritabilidad o frustración. En este mismo sentido, otro ejemplo representativo de las prácticas de gestión de la mano de obra implementadas por las plataformas es la utilización de mecanismos unilaterales de "desconexión" de trabajadores, sin previo aviso ni compensación:

"Fue en enero, tuvimos muchas huelgas, y al día siguiente de una de las huelgas, quise conectarme y recibí este mensaje: "Querido ciclista, desafortunadamente no podemos comunicarnos contigo, tu cuenta será suspendida temporalmente, te invito a que contactes con el equipo de apoyo..." (...) ;Eso no es verdad, nunca trataron de comunicarse conmigo! Envié un mail, luego otro, luego llamé, una, dos, tres veces...nunca recibí una respuesta" (Denis, repartidor). 
De manera similar, para los preparadores de pedidos, las situaciones de aislamiento social refieren directamente al hecho de que los procesos de trabajo prohíben la comunicación entre colegas, incluso en caso de mal funcionamiento del sistema de reconcomiendo vocal. A la reducción de posibilidad de comunicación entre colegas, se suma la utilización de prácticas de "performance" entre compañeros que tienden a socavar la cohesión de los colectivos de trabajo.

" iNo! no podemos hacer preguntas y no hay necesidad de hacerlas, y no es necesario hablar con el trabajador que está en la siguiente área o que viene a ayudarte, porque si hablas, pierdes líneas de productividad" (Myriam, preparadora de pedidos).

"La mayoría de los preparadores son jóvenes e inevitablemente todos están en sus cosas y el clima de trabajo ya no es bueno porque ya ni siquiera se hablan, cada uno está en lo suyo, pensando en lo que le dice el voice y en la productividad. Es más, muchas veces compiten entre ellos para ver quién hace más que el otro... eso es muy malo para todo el equipo. Ya les hemos dicho, pero es inútil..." (Robert, preparador de pedidos).

Por último, la situación de los repartidores pone de relieve la repercusión del alto nivel de rotación de los trabajadores el desarrollo a largo plazo, de un sentimiento de pertenencia a un colectivo.

“ ¿En ningún momento te van a decir, buen trabajo! Tienen tantos repartidores... es por eso que cambiaron el sistema de remuneración, ahora pueden "contratar" $y$ "despedir" al repartidor cuando quieran. ¡La rotación de los trabajadores es impresionante! Tomamos uno, si no va, lo descartamos y tomamos uno nuevo" (Víctor, repartidor).

Al alto nivel de rotación del personal, se añade la dispersión de los trabajadores por el tipo de tarea realizada, la ausencia de un espacio de trabajo físico compartido cotidianamente por los trabajadores y la progresiva reducción de otras posibilidades de intercambio. En este sentido, resulta significativo que los cambios en las condiciones de contratación introducidos a partir de 2018 hayan sido acompañados de la supresión de mecanismos susceptibles de desencadenar una dinámica colectiva de cohesión e intercambio entre los trabajadores.

"Ya no tenemos contacto con otras personas que trabajan para la plataforma. (...) Hay menos facilidad de comunicación. Al principio teníamos una lista con los nombres de los repartidores que trabajaban con nosotros. Luego, cuando 
pasamos a ser "autónomos", ya nadie sabe quién trabaja para la plataforma. Es más, antes teníamos un chat interno, ique lo suprimieron en el 2018! nos permitía organizar manifestaciones para reclamar una mejora en nuestras condiciones de trabajo (...) Además, con el nuevo sistema de pago por pedido, los repartidores ya no tienen tiempo para encontrarse en el centro de la zona para discutir" (Valter, repartidor).

En este mismo sentido apunta la intervención de otros repartidores para dar cuenta de la forma en que la aplicación es utilizada para desincentivar los espacios de encuentro y la constitución de un colectivo de trabajadores.

"En realidad, no sólo tenemos menos tiempo, sino que ahora la aplicación ya no recomienda ir al centro de la zona" (Denis, repartidor).

\section{Conclusiones}

A partir de los casos analizados, este estudio puso en evidencia el impacto de las nuevas formas de trabajo en la salud de los trabajadores. De acuerdo con nuestra perspectiva metodológica, tomó central importancia el análisis de los usos sociales de los dispositivos digitales, en el área laboral. Por lo tanto, surge en forma relevante la manera en que los cambios concretos sobre las distintas dimensiones del trabajo, tales como su organización, las condiciones, el contenido, el medioambiente y las relaciones interpersonales, se traducen en un aumento de la carga física y mental del trabajo.

En este sentido, pese a lo disímil de las actividades analizadas en el estudio actividades de reparto de mercancías, de preparación de pedidos en depósitos con el sistema picking y las relacionadas con la gestión de expedientes administrativos y bancarios, en el marco de la implementación "new ways of working"-, los resultados de la encuesta cualitativa permiten mostrar una serie de dinámicas comunes, importantes para identificar riegos laborales que se desprenden de modos de organización de trabajo flexible y del uso de dispositivos digitales.

En primer lugar, señalamos el rol determinante que juega la introducción de nuevas tecnologías, en la intensificación del trabajo, al aumentar la distancia que existe entre la tarea prescripta y el trabajo real.

En segundo lugar, destacamos la incidencia del uso de dispositivos digitales en el trabajo, sobre la disponibilidad temporal y los factores de riesgos psicosociales asociados al sentimiento de conectividad permanente. Al respecto, los diferentes casos analizados evidencian la contraposición existente entre dos situaciones; por un lado, el 
caso de los trabajadores móviles, cuyo salario se encuentra en gran medida desconectado de la medición del tiempo y por el otro, el caso de repartidores de plataformas digitales para quienes el tiempo de trabajo se contabiliza estrictamente, pero sólo se pagan las horas de trabajo efectivas (MARTINEZ, 2015).

Por último, se visualiza la problemática de la soledad, el aislamiento social, la dificultad de establecer lazos entre colegas, y por lo tanto, la desorganización en el colectivo de los trabajadores.

Finalmente, estos resultados conducen a cuestionarnos acerca de algunas de las futuras líneas de investigación que derivan de este trabajo. De hecho, a través del análisis de los riesgos laborales asociados a estas nuevas formas de trabajo en el contexto de la digitalización de la economía, en este artículo se han identificado varios obstáculos para la acción colectiva, que requieren un análisis más profundo sobre el rol de la participación de los trabajadores en la construcción de mecanismos para objetivar, visibilizar y prevenir los riegos laborales.

\section{Bibliografía}

AndonOVA, Y., "Éloge de l'indisponibilité numérique au travail. Les enjeux de l'information et de la communication", La communication numérique: acteurs, dispositifs, pratiques, $\mathrm{n}^{\circ}$ 17/3A, 2016, p. 37-48.

CAROLy, S., "Les mutations du travail face aux défis technologiques : quelles incidences sur la santé?”, Perspectives interdisciplinaires sur le travail et la santé, no 9-2, 2007 [En línea], [Fecha de consulta: 12//02/2018] Disponible en : http://journals.openedition.org/pistes/2993

DAVEZIES, P., "Enjeux de santé liés à l'utilisation de la commande vocale sur les platesformes logistiques", Enquête exploratoire, Institut Universitaire de Médecine et Santé au Travail Université Claude Bernard - Lyon 1, 2008, 48p. [En línea], [Fecha de consulta : 9/03/2018], Disponible en : http://philippe.davezies.free.fr/download/down/Voice\%20picking\%20rapport.pdf

DEGRYSE, C., Les impacts sociaux de la digitalisation de l'économie, European Trade Union Institute, 2016.

DE STEFAnO, V., The rise of the «just-in-time workforce»: on-demand work, crowdwork and labour protection in the "gig-economy», ILO, Working Paper, International Labour 
Organization, 2016, [En línea], [Fecha de consulta : 24/01/2018], Disponible en : https://econpapers.repec.org/paper/iloilowps/994899823402676.htm

DUJARIER, M-A., "Digital labor travail du consommateur, quels usages sociaux du numérique?", INA Global, La Revue des Industries Créatives et des Médias, 2016, URL :https://www.inaglobal.fr/numerique/article/digitallabor-travail-du-consommateurquels-usages-sociaux-du-numerique-8729

DURAND, J-P, La chaîne invisible. Travailler aujourd'hui : flux tendu et servitude volontaire, Éditions du Seuil, Paris, 2004.

Eurofound, New forms of employment, Publications Office of the European Union, Luxembourg, 2015.

EuROFOund, Sixth European Working Conditions Survey - Overview report (2017 update), Publications Office of the European Union Luxembourg, 2017.

EUROFOUND, Working anytime, anywhere: the effects on the world of work, Publications Office of the European Union, Luxembourg, 2017.

EUROFOUND, Overview of new forms of employment - 2018 update, Luxembourg, Publications Office of the European Union, Luxembourg, 2018.

EU-OSHA, Expert forecast on emerging psychosocial risks related to occupational safety and health. Research Report, 2007.

Gollac, M., "L'intensité du travail : formes et effets", Revue économique, $\mathrm{n}^{\circ} 2 / 56$, 2005, [En línea], [Fecha de consulta: 20/12/2017], Disponible en : https://www.cairn.info/revue-economique-2005-2-page-195.htm

Huws, U., SPEnCER N., SyRdal D. y Holts K., Work in the European Gig Economy: Research results from the UK, Sweden, Germany, Austria, the Netherlands, Switzerland and Italy, Foundation for European Progressive Studies, 2017 [En línea] [Fecha de consulta : 12/03/2018], Disponible en : https://www.feps-europe.eu/articles/36project/45-crowd-work-in-the-european-gig-economy-employment-in-the-era-of-onlineplatforms-conference-on-the-launch-of-the-report.html

Linhart, D., La comédie humaine du travail. De la déshumanisation taylorienne à la sur-humanisation managériale, Erès, coll. «Sociologie clinique», Paris, 2015. 
LAFuente HernándeZ, S., Jamar, D., Y MARTíneZ, E., “¿Qué respuestas sindicales ante el desafío de la empresa-red? Dos ejemplos de acción sindical en el sector petroquímico y nuclear", Cuadernos de Relaciones Laborales, vol. 34, n 1, 2016, p. 151.-171.

INRS (InStitut NATIONAL DE RECHERCHE ET DE SÉCURITÉ), Plateformisation 2027. Conséquences de l'ubérisation en santé et sécurité au travail, Palais du Luxembourg, Paris, 2018.

IRANI, L. C. y Silberman, M. S., "Turkopticon: Interrupting Worker Invisibility in Amazon Mechanical Turk", Proceedings of the SIGCHI Conference on Human Factors in Computing Systems, New York, USA: ACM, 2013 Disponible en : https://doi.org/10.1145/2470654.2470742

MARTINEZ, E., "La disponibilidad temporal de los asalariados en las organizaciones flexibles de trabajo", en PRIETO, C. y CRIADO, M. (Ed.), Conflictos por el tiempo. Poder, relación salarial y relaciones de género, Universidad Complutense de Madrid, CIS, 2015, p. 25-48.

Mettling, B., Transformation numérique et vie au travail, Ministre du Travail, de l'Emploi, de la Formation Professionnelle et du Dialogue Social, Paris, 2015, [En línea], [Fecha de consulta:10/03/2018], Disponible en : http://travailemploi.gouv.fr/IMG/pdf/Rapport_Mettling_Transformation_numerique_vie _au_travail.pdf

Oddone, I. y Briante, G., Redécouvrir l'expérience du travail (2è ed.). Paris: Les éditions sociales, 2015.

PoPMA, J., Technostress et autres revers du travail nomade, European Trade Union Institute, Bruxelles, 2013.

Service Public Fédéral Emploi, Travail et Concertation sociale, Guide pour la prévention des risques psychosociaux au travail, Direction générale Humanisation du Travail du Service, Bruxelles, 2017.

TASKIN, L., "La déspatialisation. Enjeu de gestion", Revue française de gestion, $\mathrm{n}^{\circ} 202$, 2010, p. 61-76.

Valenduc, G. y Vendramin, P., Le travail dans l'économie digitale: continuités et ruptures, European Trade Union Institute, Bruxelles, 2016. 
Vendramin, P. y VAlenduc, G., Le travail virtuel. Nouvelles formes de travail et d'emploi dans l'économie digitale, Fondation Travail-Université, Namur, 2016.

Fecha recepción: 17.6.2019

Fecha aceptación: 2.7.2019 